\title{
Quantum Model of Classical Mechanics: Maximum Entropy Packets
}

\section{P. Hájíček}

Received: 14 January 2009 / Accepted: 11 May 2009 / Published online: 2 June 2009

(C) Springer Science+Business Media, LLC 2009

\begin{abstract}
In a previous paper, a statistical method of constructing quantum models of classical properties has been described. The present paper concludes the description by turning to classical mechanics. The quantum states that maximize entropy for given averages and variances of coordinates and momenta are called ME packets. They generalize the Gaussian wave packets. A non-trivial extension of the partition-function method of probability calculus to quantum mechanics is given. Non-commutativity of quantum variables limits its usefulness. Still, the general form of the state operators of ME packets is obtained with its help. The diagonal representation of the operators is found. A general way of calculating averages that can replace the partition function method is described. Classical mechanics is reinterpreted as a statistical theory. Classical trajectories are replaced by classical ME packets. Quantum states approximate classical ones if the product of the coordinate and momentum variances is much larger than Planck constant. Thus, ME packets with large variances follow their classical counterparts better than Gaussian wave packets.
\end{abstract}

Keywords Classical properties · Measurement problem · Interpretation of quantum mechanics $\cdot$ Entropy $\cdot$ Partition function

\section{Introduction}

'The quantum origin of the classical' [1] is a non trivial open problem of quantum theory: 'how to explain within quantum theory the classical appearance of our macroscopic world' [2]. The purpose of the present paper is to explain the classical properties as specific properties of quantum systems. The conceptual structure of quantum

P. Hájičcek ( $\square)$

Institute for Theoretical Physics, University of Bern, Sidlerstrasse 5, 3012 Bern, Switzerland

e-mail: hajicek@itp.unibe.ch 
mechanics and with it the foundation of modern physics cannot be completely understood without such explanation.

Every existing attempt in this direction starts with the assumption that the basic properties of individual quantum systems are single values of observables and that all other properties can be constructed or derived from these basic ones. Then, quantum mechanics does not admit any genuine realist interpretation. The definitive account is given by the Bub-Clifton-Goldstein theorem [3]. Only different kinds of apparent realism for different restricted sets of properties are possible. It is then difficult to explain how objective classical properties can emerge within quantum mechanics.

In [4], we have initiated a very different approach. Our two main starting points are:

1. Value of an observable $o$ of an individual quantum system $A$ is not a property of $A$ alone, but of a composed system $A+M$, where $M$ is an apparatus measuring $o$. The value of $o$ measured by $M$ is not determined (in general) before its measurement by $M$. It is created by the measurement process. We call, therefore, single values of observables measurable on A extrinsic properties of $A$. The whole existing quantum mechanics is practically only the theory of the extrinsic properties.

2. As properties of a quantum system $A$, we allow also quantities that (a) have values that may be more complex mathematical objects than just real numbers (such as sets, mappings between sets, etc.) and (b) such a value need not be directly observable in a single measurement.

Then, there are properties of quantum systems that can be viewed as determinate before their measurements without any other condition. They have been called intrinsic, listed and classified into structural and conditional in [4]. Structural are those that are uniquely determined by the kind of quantum system (systems of the same kind are indistinguishable in the well-known strong and exclusively quantum-mechanical sense). Conditional are those that are uniquely determined by preparations. In [4], a new realist interpretation of quantum mechanics has been described based on the intrinsic properties.

Some of the intrinsic properties have been proposed as quantum models of classical properties in [4]. In particular, two kinds of conditional properties have been important: averages of quantum observables (including their variances) in a prepared state and the von-Neumann entropy of the state. Some explanatory remarks may be helpful. First, any preparation is defined by physical (objective) conditions. It need not be a process carried out by humans. Second, in the approach of [4], an average of an observable is not constructed or derived from the "more basic" single values of the observable. Averages are determined uniquely by preparation, single values are not. Not the single values determine the average but the average restrict possible single values.

Third, entropy is often considered as a measure of observer ignorance and, therefore, as a subjective concept. The "therefore" is fallacious. Entropy can generally be defined as follows. Let $\Gamma$ be a complete set of mutually exclusive properties or states of a system $A$ that is a measurable set and let the measure be $\mu(\rho)$ for $\rho \in \Gamma$. Let physical condition $C$ on $A$ lead to restriction on possible properties or states of $A$ such that the probability of the state $\rho$ to occur under $C$ is $p(\rho)$. Then the entropy $S(C)=-\int_{\Gamma} \mu(\rho) p(\rho)$. 
Such entropy can be a measure of ignorance in the following sense: everything we may know on $A$ are some physical properties that therefore define some physical condition $C$. Then the above value of entropy gives the objective uncertainty on the properties or states of the system associated with condition $C$. Thus it is, in this case, if we know only $C$, simultaneously a measure of our ignorance on the system.

Let us now briefly review the most popular approaches to the problem of classicality. At the present time, the problem does not seem to be solved in a satisfactory way, the shortcoming of the approaches being well known [5, 16, 17]. We mention them only fleetingly. First, the quantum decoherence theory $[1,2]$ works only if certain observables concerning both the environment and the quantum system cannot be measured (see the analysis in $[3,5]$ ). The deep reason is that one works with values of observables. Second, the theories based on coarse-grained operators [6-8]: the problem is the same as with the decoherence. For example, the Legget-Garg inequality [8] is a condition for the validity of the principle of macroscopic realism that works with values of observables. Third, the Coleman-Hepp theory [9-13] and its modifications $[14,15]$ : they are based on some particular theorems that hold for infinite systems but do not hold even approximately for finite ones (see the analysis in [10]).

The approach of [4] is free of these shortcomings. First, intrinsic properties are quantum properties of all quantum systems and there is no question about how they emerge in quantum mechanics. This avoids e.g. the artificial construction of classical properties in the Coleman-Hepp approach. Second, they are considered as, and proved to be, objective in [4]. Hence, second, they could in principle serve as classical properties because they can satisfy the principle of classical realism. This avoids the problems of both the quantum-decoherence and the coarse-grained theory that assume values of quantum observables to be basic properties.

More specifically, [4] has conjectured that intrinsic averages and entropy for certain macroscopic quantum systems can model all their classical properties. Classical states of a macroscopic quantum system $T$ have been defined as determined by averages $O_{1}, \ldots, O_{k}$ of quantum operators $o_{1}, \ldots, o_{k}$ that form a small subset of the algebra of observables of $T$. Finally, our modeling or construction of classical properties is nothing but statistical physics. The statistical methods that were highlighted in [4] can only work if the following hypothesis (basic hypothesis of statistical physics) is correct: The overwhelming part of macroscopic systems occur in quantum states that maximize entropy under the conditions of given averages $O_{1}, \ldots, O_{k}$. This hypothesis is supported by observation and can be derived from quantum mechanics for a class of simplified models such as [18-20].

The problem of quantum measurement (see, e.g., [5, 6, 17, 21] and references listed there) can be formulated within our approach as follows. For a measurement by a quantum apparatus $M$ on a quantum system $A$, there must be an interaction between $M$ and $A$ as well as processes in $M$ satisfying the conditions: (a) $M$ changes its classical state as the result of the interaction, (b) the change depends on the initial quantum state of $A$, (c) average values defining different resulting classical states must differ by much more than the values of their variances, and (d) Born rule is fulfilled. The knowledge of what properties of $M$ can be considered as classical is the first step. Still, to construct a model of interaction and processes in $M$ satisfying conditions (a), (b), (c) and (d) remains a non trivial problem. Ref. [4] and the present paper do not offer a solution to the problem of measurement. 
A way of model construction for internal (thermodynamic) properties of macroscopic quantum systems was described in detail in [4]. This, however, did not work for external (mechanical) properties of such systems. The aim of the present paper is to fill in this gap.

The plan of the paper is as follows. In Sect. 2, classical mechanics is interpreted as a statistical theory. The existence of sharp trajectories is rejected so that all possible states of systems are described by fuzzy distribution functions. One choice for such distributions are the so-called maximum-entropy packets (ME-packet). These are states that maximize entropy for given averages and variances of coordinates and momenta. The method of partition function is used to calculate the general form of the distribution function. For a simple solvable example, the dynamical equations for the averages and variances are obtained. The example shows how the equations of motion are reinterpreted in our theory. For general potentials, we use an approximative method: step-by-step calculation of the higher and higher time derivatives of coordinates and momenta. This will later be compared with quantum ME packets.

Turning to quantum mechanics, we apply the maximum-entropy principle in an analogous way in Sect. 3. The averages and variances are taken over from the classical states that are to be modeled. A straightforward generalization of the partition function method is now complicated by the non-commutativity of the coordinates and momenta. We can show that only the first derivatives of the logarithm of partition function have the usual meaning. This is, however, sufficient for calculating the state operators for all ME packets. We find the diagonal representation of the state operator in Sect. 3.2 and obtain with its help the general form of the partition function and the state operator itself. It turns out that Gaussian wave packets are special case of ME packets, namely those with zero entropy and minimal uncertainty. The diagonal representation gives us also a powerful method to calculate averages of higher moments. In fact, what has been done in Sects. 3.1 and 3.2 is a non-trivial extension of the partition-function method of the probability calculus as described, e.g., in [22] to quantum mechanics that might also be of some interest for mathematicians.

In Sect. 3.3, the equations of motion are calculated in analogy to the classical case. We find that the quantum corrections to the classical equations come only from high powers of $q$ in the expansion of the potential or in high powers of $t$ in the expansion of the time-dependent averages. Also, these corrections are of the second order in $\hbar$. These results show that our quantum models follows classical trajectories very closely. The nature of classical limit is studied in Sect. 4 . The result, which may seem surprising, is that it is the limit of large variances, not small. Thus, quantum ME packets with large variances follow their classical counterparts better than Gaussian wave packets. Of course, the way we measure the size of the variances is important here. The variances that are large with respect to this measure can still be sufficiently small to agree with observations. Finally, Sect. 5 concludes the paper by summarizing the main ideas and the most important results.

\section{Statistical form of Classical Mechanics}

Let us start with the warning that the topic of this section has nothing to do with what is usually called 'statistical mechanics'. 
If one is going to model classical mechanics then what are the properties that one would like to reproduce? The most conspicuous property from the point of view of quantum mechanics appears to be the sharpness of mechanical trajectories in the phase space because quantum mechanics denies the existence of such trajectories. This leads most researchers to aim at quantum states the phase-space picture of which is as sharp as possible. That are states with minimum uncertainty allowed by quantum mechanics. For one degree of freedom, described by coordinate $q$ and momentum $p$, the uncertainty is given by the quantity

$$
v=\frac{2 \Delta q \Delta p}{\hbar}
$$

where $\Delta a$ is the variance of quantity $a$,

$$
\Delta a=\sqrt{\left\langle a^{2}\right\rangle-\langle a\rangle^{2}} .
$$

It is well known that minimum uncertainty allowed by quantum mechanics is $v=1$.

The states with $v=1$ are, however, very special states. First, they must be pure states such as Gaussian wave packets or coherent states. Such states are very difficult to prepare unlike the usual states of macroscopic systems described by classical mechanics. They are also prone to strong distortion by measurements. Moreover, as pure states, they can be linearly superposed. This is another peculiarity that is never observed for states of systems of classical mechanics. Hence, trying to get a trajectory as sharp as possible leads to the loss of other desirable properties.

Moreover, observations within classical mechanics admit the notion that the sharpness of phase-space trajectories is only a mathematical and methodical feature of classical mechanics. It may be just an idealization, a limit in which things become mathematically simpler. We can use it in calculations which, however, must also take into account the necessary non-zero variances of real observations. Indeed, such observations are generally afflicted with uncertainties $v \gg 1$. Hence, if we want to compare the predictions of our quantum models with observations of classical mechanics, we are forced to compare states that are fuzzy in both theories.

One idea of the present paper is to consider states with given averages and variances of the coordinates and momenta and leave everything else as fuzzy as possible. To calculate the corresponding probability distributions in classical, and the state operators in quantum mechanics, we shall, therefore, apply the maximum entropy principle. This is a general principle in mathematical theory of probabilities (see [22]) and it should not be confused with the well-known thermodynamic law. The resulting states are called maximum-entropy packets, ME-packets. The averages of coordinates and momenta take over the role of coordinate and momenta in classical mechanics. In any case the averages represent measurable aspects of these variables. The dynamical evolution of variances is an important indicator of the applicability of the model one is working with. It determines the time intervals within which reasonable predictions are possible.

Consider a three-body system that is to model the Sun, Earth and Jupiter. It turns out that generic trajectories starting as near to each other as, say, the dimension of the irregularities of the Earth surface will diverge from each other by dimensions of 
the Earth-Sun distance after the time of only about ten million years. This seems to contradict the four billion years of relatively stable Earth motion around the Sun that is born out by observations. The only way out is the existence of a few special trajectories that are much stabler than the generic ones and the fact that bodies following an unstable trajectory have long ago fallen into the Sun or have been ejected from the solar system.

An important question is that on the ontological status of ME-packets and on the nature of the limit in which trajectories become sharp. The usual standpoint is that any mechanical system always objectively is in a state of a completely sharp trajectory. Any more fuzzy state is only the result of our incomplete knowledge. Thus, the fuzzy states are not considered themselves as real. Here, we take the opposite standpoint. For us, a state to be real, it must be determined by objective initial conditions. A simple example is a gun in a position that is fixed in a reproducible way and that shoot bullets using cartridges of a given provenance. The state of each individual shot is defined by the conditions and is the same for all shots even if observations may have different results for different shots. A finer analysis is possible only as long as new initial conditions are specified that determine a subset of individual shots. In the theoretical description of a state, we can make the limit of $\Delta Q \rightarrow 0, \Delta P \rightarrow 0$. This is considered as a non-existing, but practically useful idealization.

To limit ourselves just to given averages and variances of coordinates and momenta is a great simplification that enables us to obtain interesting results easily. Some further discussion on quantum modeling of classical properties is in order. On the one hand, for internal degrees of freedom, the usual thermodynamic methods give small relative variances as a consequence of the state coordinates being extensive, the entropy being maximal and the system being macroscopic. This does not work for external (mechanical) properties. The difference is due to the simple fact that the internal degrees of freedom are not accessible to manipulation and have small variances spontaneously. The external degrees of freedom are accessible to manipulations and it is easy to prepare states with small as well as large variances. There is no objective need that the variances are small spontaneously. The idea that really existing mechanical states must always have only small variances is caused by a purely theoretical notion that all real mechanical systems have an absolutely sharp phase space trajectory and this notion is clearly false. The only problem is that it has become a part of our subconscious psychology.

On the other hand, if quantum ME packets are to be quantum models of the classical ME packets with the same averages and variances then this is a more general situation than that considered in Ref. [4]. Quantum ME packet is a classical state in the sense of Ref. [4] if it has small variances. Only then, the average values are directly observable on individual systems.

\subsection{Classical ME-Packets}

Let us first consider systems with one degree of freedom. The generalization to any number is easy. Let the coordinate be $q$ and the momentum $p$. A state is a distribution function $\rho(q, p)$ on the phase space spanned by $q$ and $p$. The function $\rho(q, p)$ is 
dimension-free and normalized by

$$
\int \frac{d q d p}{v} \rho=1
$$

where $v$ is an auxiliary phase-space volume to make the integration dimension-free. The entropy of $\rho(q, p)$ can be defined by

$$
S:=-\int \frac{d q d p}{v} \rho \ln \rho .
$$

The value of entropy will depend on $v$ but the most of other results will not. Classical mechanics does not offer any idea of how to fix $v$. We shall get its value from quantum mechanics.

Let us define: ME-packet is the distribution function $\rho$ that maximizes the entropy subjected to the conditions:

$$
\langle q\rangle=Q, \quad\left\langle q^{2}\right\rangle=\Delta Q^{2}+Q^{2},
$$

and

$$
\langle p\rangle=P, \quad\left\langle p^{2}\right\rangle=\Delta P^{2}+P^{2},
$$

where $Q, P, \Delta Q$ and $\Delta P$ are given values of averages and variances of $q$ and $p$. We have used the abbreviation

$$
\langle x\rangle=\int \frac{d q d p}{v} x .
$$

The explicit form of $\rho$ can be found using the partition-function method as described e.g. in [22]. The variational principle yields

$$
\rho=\frac{1}{Z\left(\lambda_{1}, \lambda_{2}, \lambda_{3}, \lambda_{4}\right)} \exp \left(-\lambda_{1} q-\lambda_{2} p-\lambda_{3} q^{2}-\lambda_{4} p^{2}\right),
$$

where

$$
Z=\int \frac{d q d p}{v} \exp \left(-\lambda_{1} q-\lambda_{2} p-\lambda_{3} q^{2}-\lambda_{4} p^{2}\right),
$$

and $\lambda_{1}, \lambda_{2}, \lambda_{3}$ and $\lambda_{4}$ are the Lagrange multipliers. Hence, the partition function for classical ME-packets is given by

$$
Z=\frac{\pi}{v} \frac{1}{\sqrt{\lambda_{3} \lambda_{4}}} \exp \left(\frac{\lambda_{1}^{2}}{4 \lambda_{3}}+\frac{\lambda_{2}^{2}}{4 \lambda_{4}}\right) .
$$

The expressions for $\lambda_{1}, \lambda_{2}, \lambda_{3}$ and $\lambda_{4}$ in terms of $Q, P, \Delta Q$ and $\Delta P$ can be obtained by solving the equations

$$
\frac{\partial \ln Z}{\partial \lambda_{1}}=-Q, \quad \frac{\partial \ln Z}{\partial \lambda_{3}}=-\Delta Q^{2}-Q^{2},
$$


and

$$
\frac{\partial \ln Z}{\partial \lambda_{2}}=-P, \quad \frac{\partial \ln Z}{\partial \lambda_{4}}=-\Delta P^{2}-P^{2} .
$$

The result is:

$$
\lambda_{1}=-\frac{Q}{\Delta Q^{2}}, \quad \lambda_{3}=\frac{1}{2 \Delta Q^{2}},
$$

and

$$
\lambda_{2}=-\frac{P}{\Delta P^{2}}, \quad \lambda_{4}=\frac{1}{2 \Delta P^{2}} .
$$

Substituting this into (5), we obtain the distribution function of a one-dimensional ME packet. The generalization to any number of dimensions is trivial.

Theorem 1 The distribution function of the ME-packet for a system with given averages and variances $Q_{1}, \ldots, Q_{n}, \Delta Q_{1}, \ldots, \Delta Q_{n}$ of coordinates and $P_{1}, \ldots, P_{n}$, $\Delta P_{1}, \ldots, \Delta P_{n}$ of momenta, is

$$
\rho=\left(\frac{v}{2 \pi}\right)^{n} \prod_{k=1}^{n}\left(\frac{1}{\Delta Q_{k} \Delta P_{k}} \exp \left[-\frac{\left(q_{k}-Q_{k}\right)^{2}}{2 \Delta Q_{k}^{2}}-\frac{\left(p_{k}-P_{k}\right)^{2}}{2 \Delta P_{k}^{2}}\right]\right) .
$$

We observe that all averages obtained from $\rho$ are independent of $v$ and that the result is a Gaussian distribution in agreement with Jaynes' conjecture that the maximum entropy principle gives the Gaussian distribution if the only conditions are fixed values of the first two moments.

As $\Delta Q$ and $\Delta P$ approach zero, $\rho$ becomes a delta-function and the state becomes sharp. For some quantities, this limit is sensible for others it is not. In particular, the entropy, which can easily be calculated,

$$
S=1+\ln \frac{2 \pi \Delta Q \Delta P}{v},
$$

diverges to $-\infty$. This is due to a general difficulty in giving a definition of entropy for a continuous system that would be satisfactory in every respect. What one could do is to divide the phase space into cells of volume $v$ so that $\Delta Q \Delta P$ could not be chosen smaller than $v$. Then, the limit $\Delta Q \Delta P \rightarrow v$ of entropy would make more sense.

The average of any monomial of the form $q^{k} p^{l} q^{2 m} p^{2 n}$ can be calculated with the help of partition-function method as follows:

$$
\left\langle q^{k} p^{l} q^{2 m} p^{2 n}\right\rangle=\frac{(-1)^{\mathbf{N}}}{Z} \frac{\partial^{\mathbf{N}} Z}{\partial \lambda_{1}^{k} \partial \lambda_{2}^{l} \partial \lambda_{3}^{m} \partial \lambda_{4}^{n}},
$$

where $\mathbf{N}=k+l+2 m+2 n, Z$ is given by (6) and the values (7) and (8) must be substituted for the Lagrange multipliers after the derivatives are taken. 
Observe that this enables to calculate the average of a monomial in several different ways. Each of these ways, however, leads to the same result due the identities

$$
\frac{\partial^{2} Z}{\partial \lambda_{1}^{2}}=-\frac{\partial Z}{\partial \lambda_{3}}, \quad \frac{\partial^{2} Z}{\partial \lambda_{2}^{2}}=-\frac{\partial Z}{\partial \lambda_{4}},
$$

which are satisfied by the partition function.

\subsection{Equations of Motion}

Let us assume that the Hamiltonian of our system has the form

$$
H=\frac{p^{2}}{2 m}+V(q),
$$

where $m$ is the mass and $V(q)$ the potential function. The equations of motion are

$$
\dot{q}=\{q, H\}, \quad \dot{p}=\{p, H\} .
$$

Inserting (11) for $H$, we obtain

$$
\dot{q}=\frac{p}{m}, \quad \dot{p}=-\frac{d V}{d q} .
$$

The general solution to these equations can be written in the form

$$
q(t)=q(t ; q, p), \quad p(t)=p(t ; q, p),
$$

where

$$
q(0 ; q, p)=q, \quad p(0 ; q, p)=p,
$$

$q$ and $p$ being arbitrary initial values. We obtain the equations of motion for the averages and variances:

$$
Q(t)=\langle q(t ; q, p)\rangle, \quad \Delta Q(t)=\sqrt{\left\langle(q(t ; q, p)-Q(t))^{2}\right\rangle}
$$

and

$$
P(t)=\langle p(t ; q, p)\rangle, \quad \Delta P(t)=\sqrt{\left\langle(p(t ; q, p)-P(t))^{2}\right\rangle} .
$$

In general, $Q(t)$ and $P(t)$ will depend not only on $Q$ and $P$, but also on $\Delta Q$ and $\Delta P$.

Let us consider the special case of at most quadratic potential:

$$
V(q)=V_{0}+V_{1} q+\frac{1}{2} V_{2} q^{2}
$$

where $V_{k}$ are constants with suitable dimensions. If $V_{1}=V_{2}=0$, we have a free particle, if $V_{2}=0$, it is a particle in a homogeneous force field and if $V_{2} \neq 0$, it is an harmonic or anti-harmonic oscillator. 
In this case, the general solution has the form

$$
\begin{aligned}
& q(t)=f_{0}(t)+q f_{1}(t)+p f_{2}(t), \\
& p(t)=g_{0}(t)+q g_{1}(t)+p g_{2}(t),
\end{aligned}
$$

where $f_{0}(0)=f_{2}(0)=g_{0}(0)=g_{1}(0)=0$ and $f_{1}(0)=g_{2}(0)=1$. If $V_{2} \neq 0$, the functions are

$$
\begin{aligned}
& f_{0}(t)=-\frac{V_{1}}{V_{2}}(1-\cos \omega t), \quad f_{1}(t)=\cos \omega t, \quad f_{2}(t)=\frac{1}{\xi} \sin \omega t, \\
& g_{0}(t)=-\xi \frac{V_{1}}{V_{2}} \sin \omega t, \quad g_{1}(t)=-\xi \sin \omega t, \quad g_{2}(t)=\cos \omega t,
\end{aligned}
$$

where

$$
\xi=\sqrt{m V_{2}}, \quad \omega=\sqrt{\frac{V_{2}}{m}} .
$$

Only for $V_{2}>0$, the functions remain bounded. If $V_{2}=0$, we obtain

$$
\begin{array}{lrl}
f_{0}(t)=-\frac{V_{1}}{2 m} t^{2}, & f_{1}(t)=1, & f_{2}(t)=\frac{t}{m}, \\
g_{0}(t)=-V_{1} t, & g_{1}(t)=0, & g_{2}(t)=1 .
\end{array}
$$

The equations for averages and variances resulting from (13), (3) and (4) are

$$
Q(t)=f_{0}(t)+Q f_{1}(t)+P f_{2}(t),
$$

and

$$
\begin{aligned}
\Delta Q^{2}(t)+Q^{2}(t)= & f_{0}^{2}(t)+\left(\Delta Q^{2}+Q^{2}\right) f_{1}^{2}(t)+\left(\Delta P^{2}+P^{2}\right) f_{2}^{2}(t) \\
& +2 Q f_{0}(t) f_{1}(t)+2 P f_{0}(t) f_{2}(t)+2\langle q p\rangle f_{1}(t) f_{2}(t) .
\end{aligned}
$$

For the last term, we have from (10)

$$
\langle q p\rangle=\frac{1}{Z} \frac{\partial^{2} Z}{\partial \lambda_{1} \partial \lambda_{2}} .
$$

Using (6), (7) and (8), we obtain from (25)

$$
\Delta Q(t)=\sqrt{f_{1}^{2}(t) \Delta Q^{2}+f_{2}^{2}(t) \Delta P^{2}} .
$$

Similarly,

$$
\begin{gathered}
P(t)=g_{0}(t)+Q g_{1}(t)+P g_{2}(t), \\
\Delta P(t)=\sqrt{f_{g}^{2}(t) \Delta Q^{2}+g_{2}^{2}(t) \Delta P^{2}} .
\end{gathered}
$$

We observe: if functions $f_{1}(t), f_{2}(t), g_{1}(t)$ and $g_{2}(t)$ remain bounded, the variances also remain bounded and the predictions are possible in arbitrary long intervals of 
time. Otherwise, there will always be only limited time intervals in which the theory can make predictions.

In the case of general potential, the functions (13) can be expanded in products of powers of $q$ and $p$, and the averages of these products will contain powers of the variances. However, as one easily sees form formula (10) and (6),

$$
\left\langle q^{k} p^{l}\right\rangle=Q^{k} P^{l}+X \Delta Q+Y \Delta P,
$$

where $X$ and $Y$ are bounded functions. It follows that the dynamical equations for averages coincide, in the limit $\Delta Q \rightarrow 0, \Delta P \rightarrow 0$, with the exact dynamical equations for $q$ and $p$. It is an idealization that we consider as not realistic, even in principle, but that may still be useful for calculations.

Let us expand a general potential function in powers of $q$,

$$
V(q)=\sum_{k=0}^{\infty} \frac{1}{k !} V_{k} q^{k},
$$

where $V_{k}$ are constants of appropriate dimensions. The Hamilton equations can be used to calculate all time derivatives at $t=0$. First, we have

$$
\frac{d q}{d t}=\{q, H\}=\frac{p}{m}
$$

This equation can be used to calculate all derivatives of $q$ in terms of those of $p$ :

$$
\frac{d^{n} q}{d t^{n}}=\frac{1}{m} \frac{d^{n-1} p}{d t^{n-1}} .
$$

A simple iterative procedure gives us further time derivatives of $p$ :

$$
\begin{aligned}
\frac{d p}{d t}= & -V_{1}-V_{2} q-\frac{V_{3}}{2} q^{2}-\frac{V_{4}}{6} q^{3}+r_{5}, \\
\frac{d^{2} p}{d t^{2}}= & -\frac{V_{2}}{m} p-\frac{V_{3}}{m} q p-\frac{V_{4}}{2 m} q^{2} p+r_{5}, \\
\frac{d^{3} p}{d t^{3}}= & -\frac{V_{3}}{m^{2}} p^{2}-\frac{V_{4}}{m^{2}} q p^{2}+\frac{V_{1} V_{2}}{m}+\frac{V_{1} V_{3}+V_{2}^{2}}{m} q \\
& +\frac{3 V_{2} V_{3}+V_{1} V_{4}}{2 m} q^{2}+\frac{4 V_{2} V_{4}+3 V_{3}^{2}}{6 m} q^{3}+\frac{5 V_{3} V_{4}}{12 m} q^{4}+\frac{V_{4}^{2}}{12 m} q^{5}+r_{5},
\end{aligned}
$$

and

$$
\begin{aligned}
\frac{d^{4} p}{d t^{4}}= & -\frac{V_{4}}{m^{3}} p^{3}+\frac{3 V_{1} V_{3}+V_{2}^{2}}{m^{2}} p+\frac{3 V_{1} V_{4}+5 V_{2} V_{3}}{m^{2}} q p \\
& +\frac{5 V_{3}^{2}+8 V_{2} V_{4}}{2 m^{2}} q^{2} p+3 \frac{V_{3} V_{4}}{m^{2}} q^{3} p+\frac{3 V_{4}^{2}}{4 m^{2}} q^{4} p+r_{5},
\end{aligned}
$$


where $r_{k}$ is the rest term that is due to all powers in (29) that are not smaller than $k$ (the rests symbolize different expressions in different equations). The purpose of having all time derivatives up to the fourth order is to show later that it is the highest order in which no quantum corrections appear in the equations for the averages.

Taking the average of both sides of (31)-(34), and using (10), (6)-(8), we obtain

$$
\begin{aligned}
\frac{d P}{d t}= & -V_{1}-V_{2} Q-\frac{V_{3}}{2} Q^{2}-\frac{V_{4}}{6} Q^{3}-\frac{V_{3}+V_{4} Q}{2} \Delta Q^{2}+r_{5} \\
\frac{d^{2} P}{d t^{2}}= & -\frac{V_{2}}{m} P+\frac{V_{3}}{m} Q P+\frac{V_{4}}{2 m} Q^{2} P+\frac{V_{4}}{2 m} P \Delta Q^{2}+r_{5} \\
\frac{d^{3} P}{d t^{3}}= & -\frac{V_{3}}{m^{2}} P^{2}-\frac{V_{4}}{m^{2}} Q P^{2}+\frac{V_{1} V_{2}}{m}+\frac{V_{1} V_{3}+V_{2}^{2}}{m} Q+\frac{3 V_{2} V_{3}+V_{1} V_{4}}{2 m} Q^{2} \\
& +\frac{4 V_{2} V_{4}+3 V_{3}^{2}}{6 m} Q^{3}+\frac{5 V_{3} V_{4}}{12 m} Q^{4}+\frac{V_{4}^{2}}{12 m} Q^{5}-\left(\frac{V_{3}}{m^{2}}+\frac{V_{4}}{m^{2}} Q\right) \Delta P^{2} \\
& +\left(\frac{3 V_{2} V_{3}+V_{1} V_{4}}{2 m}+\frac{4 V_{2} V_{4}+3 V_{3}^{2}}{2 m} Q+\frac{5 V_{3} V_{4}}{2 m} Q^{2}+\frac{5 V_{3} V_{4}}{4 m} \Delta Q^{2}\right. \\
& \left.+\frac{5 V_{4}^{2}}{6 m} Q^{3}+\frac{5 V_{4}^{2}}{4 m} Q \Delta Q^{2}\right) \Delta Q^{2}+r_{5},
\end{aligned}
$$

and

$$
\begin{aligned}
\frac{d^{4} P}{d t^{4}}= & -\frac{V_{4}}{m^{3}} P^{3}+\frac{3 V_{1} V_{3}+V_{2}^{2}}{m^{2}} P+\frac{3 V_{1} V_{4}+5 V_{2} V_{3}}{m^{2}} Q P \\
& +\frac{5 V_{3}^{2}+8 V_{2} V_{4}}{2 m^{2}} Q^{2} P+3 \frac{V_{3} V_{4}}{m^{2}} Q^{3} P+\frac{3 V_{4}^{2}}{4 m^{2}} Q^{4} P-\frac{3 V_{4}}{m^{3}} P \Delta P^{2} \\
& +\left(\frac{5 V_{3}^{2}+8 V_{2} V_{4}}{2 m^{2}} P+\frac{9 V_{3} V_{4}}{m^{2}} Q P+\frac{9 V_{4}^{2}}{2 m^{2}} Q^{2} P+\frac{9 V_{4}^{2}}{4 m^{2}} P \Delta Q^{2}\right) \Delta Q^{2}+r_{5} .
\end{aligned}
$$

We can see, that the limit $\Delta Q \rightarrow 0, \Delta P \rightarrow 0$ in (35)-(38) lead to equations that coincide with (31)-(34) if $Q \rightarrow q, P \rightarrow p$ as promised.

\section{Quantum ME-Packets}

Let us now turn to quantum mechanics and try to solve an analogous problem. Let a system with one degree of freedom be described by the operators $q$ and $p$ and let us look for a state $\rho$, a normalized,

$$
\operatorname{Tr} \rho=1,
$$

self-adjoint positive operator, that maximizes von Neumann entropy

$$
S=\operatorname{Tr}(\rho \ln \rho)
$$


under the conditions

$$
\begin{array}{ll}
\operatorname{Tr}(\rho q)=Q, & \operatorname{Tr}\left(\rho q^{2}\right)=Q^{2}+\Delta Q^{2}, \\
\operatorname{Tr}(\rho p)=P, & \operatorname{Tr}\left(\rho p^{2}\right)=P^{2}+\Delta P^{2},
\end{array}
$$

where $Q, P, \Delta Q$ and $\Delta P$ are given numbers. The states that satisfy these conditions are called quantum ME-packets.

\subsection{Calculation of the State Operator}

To solve the mathematical problem, we use the method of Lagrange multipliers as in the classical case. Thus, the following equation results:

$$
\begin{gathered}
d S-\lambda_{0} d \operatorname{Tr} \rho-\lambda_{1} d \operatorname{Tr}(\rho q)-\lambda_{2} d \operatorname{Tr}(\rho p) \\
-\lambda_{3} d \operatorname{Tr}\left(\rho q^{2}\right)-\lambda_{4} d \operatorname{Tr}\left(\rho p^{2}\right)=0 .
\end{gathered}
$$

The differentials of the terms that are linear in $\rho$ are simple to calculate:

$$
d \operatorname{Tr}(\rho x)=\sum_{m n} x_{n m} d \rho_{m n}
$$

Although not all elements of the matrix $d \rho_{m n}$ are independent (it is a hermitian matrix), we can proceed as if they were because the matrix $x_{n m}$ is to be also hermitian. The only problem is to calculate $d S$. We have the following

\section{Lemma 1}

$$
d S=-\sum_{m n}\left[\delta_{m n}+(\ln \rho)_{m n}\right] d \rho_{m n}
$$

Proof Let $M$ be a unitary matrix that diagonalizes $\rho$,

$$
M^{\dagger} \rho M=R,
$$

where $R$ is a diagonal matrix with elements $R_{n}$. Then $S=-\sum_{n} R_{n} \ln R_{n}$. Correction to $R_{n}$ if $\rho \mapsto \rho+d \rho$ can be calculated by the first-order formula of the stationary perturbation theory. This theory is usually applied to Hamiltonians but it holds for any perturbed hermitian operator. Moreover, the formula is exact for infinitesimal perturbations. Thus,

$$
R_{n} \mapsto R_{n}+\sum_{k l} M_{k n}^{\dagger} M_{l n} d \rho_{k l}
$$

In this way, we obtain

$$
\begin{aligned}
d S= & -\sum_{n}\left(R_{n}+\sum_{k l} M_{k n}^{\dagger} M_{l n} d \rho_{k l}\right) \\
& \times \ln \left[R_{n}\left(1+\frac{1}{R_{n}} \sum_{r s} M_{r n}^{\dagger} M_{s n} d \rho_{r s}\right)\right]-\sum_{n} R_{n} \ln R_{n}
\end{aligned}
$$




$$
\begin{aligned}
& =-\sum_{n}\left[\ln R_{n} \sum_{k l} M_{k n}^{\dagger} M_{l n} d \rho_{k l}+\sum_{k l} M_{k n}^{\dagger} M_{l n} d \rho_{k l}\right] \\
& \left.=-\sum_{k l}\left[\delta_{k l}+(\ln \rho)_{k l}\right]\right] d \rho_{k l} .
\end{aligned}
$$

With the help of Lemma 1, (42) becomes

$$
\operatorname{Tr}\left[\left(1+\ln \rho-\lambda_{0}-\lambda_{1} q-\lambda_{2} p-\lambda_{3} q^{2}-\lambda_{4} p^{2}\right) d \rho\right]=0
$$

so that we have

$$
\rho=\exp \left(-\lambda_{0}-1-\lambda_{1} q-\lambda_{2} p-\lambda_{3} q^{2}-\lambda_{4} p^{2}\right) .
$$

The first two terms in the exponent determine the normalization constant

$$
e^{-\lambda_{0}-1}
$$

because they commute with the rest of the exponent and are independent of the dynamical variables. Taking the trace of (44), we obtain

$$
e^{-\lambda_{0}-1}=\frac{1}{Z\left(\lambda_{1}, \lambda_{2}, \lambda_{3}, \lambda_{4}\right)},
$$

where $Z$ is the partition function,

$$
Z\left(\lambda_{1}, \lambda_{2}, \lambda_{3}, \lambda_{4}\right)=\operatorname{Tr}\left[\exp \left(-\lambda_{1} q-\lambda_{2} p-\lambda_{3} q^{2}-\lambda_{4} p^{2}\right)\right]
$$

Thus, the state operator has the form

$$
\rho=\frac{1}{Z\left(\lambda_{1}, \lambda_{2}, \lambda_{3}, \lambda_{4}\right)} \exp \left(-\lambda_{1} q-\lambda_{2} p-\lambda_{3} q^{2}-\lambda_{4} p^{2}\right) .
$$

At this stage, the quantum theory begins to differ from the classical one. It turns out that, for the case of non-commuting operators in the exponent of the partition function, formula (10) is not valid in general. We can only show that it holds for the first derivatives. To this aim, we prove the following

Lemma 2 Let $A$ and $B$ be Hermitian matrices. Then

$$
\frac{d}{d \lambda} \operatorname{Tr}[\exp (A+B \lambda)]=\operatorname{Tr}[B \exp (A+B \lambda)] .
$$

Proof We express the exponential function as a series and then use the invariance of trace with respect to any cyclic permutation of its argument.

$$
d \operatorname{Tr}[\exp (A+B \lambda)]=\sum_{n=0}^{\infty} \frac{1}{n !} \operatorname{Tr}\left[d(A+B \lambda)^{n}\right]
$$




$$
\begin{aligned}
& =\sum_{n=0}^{\infty} \frac{1}{n !} \operatorname{Tr}\left[\sum_{k=1}^{n}(A+B \lambda)^{k-1} B(A+B \lambda)^{n-k}\right] d \lambda \\
& =\sum_{n=0}^{\infty} \frac{1}{n !} \sum_{k=1}^{n} \operatorname{Tr}\left[B(A+B \lambda)^{n-1}\right] d \lambda \\
& =\operatorname{Tr}[B \exp (A+B \lambda)] d \lambda .
\end{aligned}
$$

The proof of Lemma 2 shows why formula (10) is not valid for higher derivatives than the first in the quantum case: the operator $B$ does not commute with $A+B \lambda$ and cannot be shifted from its position to the first position in product

$$
(A+B \lambda)^{k} B(A+B \lambda)^{l} .
$$

For the first derivative, it can be brought there by a suitable cyclic permutation. However, each commutator $[B,(A+B \lambda)]$ is proportional to $\hbar$. Hence, formula (10) with higher derivatives is the leading term in the expansion of averages in powers of $\hbar$.

Together with (45), Lemma 2 implies the formulae:

$$
\frac{\partial \ln Z}{\partial \lambda_{1}}=-Q, \quad \frac{\partial \ln Z}{\partial \lambda_{3}}=-Q^{2}-\Delta Q^{2}
$$

and

$$
\frac{\partial \ln Z}{\partial \lambda_{2}}=-P, \quad \frac{\partial \ln Z}{\partial \lambda_{4}}=-P^{2}-\Delta P^{2} .
$$

The values of the multipliers can be calculated from (48) and (49), if the form of the partition function is known.

Variational methods can find locally extremal values that are not necessarily maxima. We can however prove that our state operator maximizes entropy. The proof is based on the generalized Gibbs' inequality,

$$
\operatorname{Tr}(\rho \ln \rho-\rho \ln \sigma) \geq 0
$$

for all pairs $\{\rho, \sigma\}$ of state operators (for proof of the inequality, see [6], p. 264). The proof of maximality is then analogous to the 'classical' proof (see, e.g., [22], p. 357). The first proof of maximality in the quantum case was given by von Neumann [23].

The state operator (46) can be inserted in the formula (39) to give the value of the maximal entropy,

$$
S=\ln Z+\lambda_{1}\langle q\rangle+\lambda_{2}\langle p\rangle+\lambda_{3}\left\langle q^{2}\right\rangle+\lambda_{4}\left\langle p^{2}\right\rangle .
$$

This, together with (48) and (49) can be considered as the Legendre transformation from the function $\ln Z\left(\lambda_{1}, \lambda_{2}, \lambda_{3}, \lambda_{4}\right)$ to the function $S\left(\langle q\rangle,\langle p\rangle,\left\langle q^{2}\right\rangle,\left\langle p^{2}\right\rangle\right)$.

\subsection{Diagonal Representation}

The exponent in (46) can be written in the form

$$
\frac{\lambda_{1}^{2}}{4 \lambda_{3}}+\frac{\lambda_{2}^{2}}{4 \lambda_{4}}-2 \sqrt{\lambda_{3} \lambda_{4}} K
$$


where

$$
K=\frac{1}{2} \sqrt{\frac{\lambda_{3}}{\lambda_{4}}}\left(q+\frac{\lambda_{1}}{2 \lambda_{3}}\right)^{2}+\frac{1}{2} \sqrt{\frac{\lambda_{4}}{\lambda_{3}}}\left(p+\frac{\lambda_{2}}{2 \lambda_{4}}\right)^{2} .
$$

This is an operator acting on the Hilbert space of our system. $K$ has the form of the Hamiltonian $^{1}$ of a harmonic oscillator with the coordinate $U$ and momentum $W$

$$
U=q+\frac{\lambda_{1}}{2 \lambda_{3}}, \quad W=p+\frac{\lambda_{2}}{2 \lambda_{4}},
$$

that satisfy the commutation relation $[U, W]=i \hbar$. The oscillator has mass $M$ and frequency $\Omega$,

$$
M=\sqrt{\frac{\lambda_{3}}{\lambda_{4}}}, \quad \Omega=1 .
$$

The normalized eigenstates $|k\rangle$ of the operator form a basis in the Hilbert space of our system defining the so-called diagonal representation and its eigenvalues are $\hbar / 2+$ $\hbar k$. As usual, we introduce operator $A$ such that

$$
\begin{aligned}
U & =\sqrt{\frac{\hbar}{2 M}}\left(A+A^{\dagger}\right), \\
W & =-i \sqrt{\frac{\hbar M}{2}}\left(A-A^{\dagger}\right), \\
K & =\frac{\hbar}{2}\left(A^{\dagger} A+A A^{\dagger}\right), \\
A|k\rangle & =\sqrt{k}|k-1\rangle, \\
A^{\dagger}|k\rangle & =\sqrt{k+1}|k+1\rangle .
\end{aligned}
$$

To calculate $Z$ in the diagonal representation is easy:

$$
\begin{aligned}
Z & =\operatorname{Tr}\left[\exp \left(\frac{\lambda_{1}^{2}}{4 \lambda_{3}}+\frac{\lambda_{2}^{2}}{4 \lambda_{4}}-2 \sqrt{\lambda_{3} \lambda_{4}} K\right)\right] \\
& =\sum_{k=0}^{\infty}\left\langle k\left|\exp \left(\frac{\lambda_{1}^{2}}{4 \lambda_{3}}+\frac{\lambda_{2}^{2}}{4 \lambda_{4}}-2 \sqrt{\lambda_{3} \lambda_{4}} K\right)\right| k\right\rangle \\
& =\exp \left(\frac{\lambda_{1}^{2}}{4 \lambda_{3}}+\frac{\lambda_{2}^{2}}{4 \lambda_{4}}-\hbar \sqrt{\lambda_{3} \lambda_{4}}\right) \sum_{k=0}^{\infty} \exp \left(-2 \hbar \sqrt{\lambda_{3} \lambda_{4}} k\right) .
\end{aligned}
$$

Hence, the partition function for the quantum ME-packets is

$$
Z=\frac{\exp \left(\frac{\lambda_{1}^{2}}{4 \lambda_{3}}+\frac{\lambda_{2}^{2}}{4 \lambda_{4}}\right)}{2 \sinh \left(\hbar \sqrt{\lambda_{3} \lambda_{4}}\right)}
$$

\footnotetext{
${ }^{1}$ The operator $K$ must not be confused with the Hamiltonian $H$ of our system, which can be arbitrary.
} 
Now, we can express the Lagrange multipliers in terms of the averages and variances. Equations (48) and (49) yield

$$
\lambda_{1}=-\frac{Q}{\Delta Q^{2}} \frac{v}{2} \ln \frac{v+1}{v-1}, \quad \lambda_{2}=-\frac{P}{\Delta P^{2}} \frac{v}{2} \ln \frac{v+1}{v-1},
$$

and

$$
\lambda_{3}=\frac{1}{2 \Delta Q^{2}} \frac{v}{2} \ln \frac{v+1}{v-1}, \quad \lambda_{4}=\frac{1}{2 \Delta P^{2}} \frac{v}{2} \ln \frac{v+1}{v-1},
$$

where $v$ is defined by (1).

From (50), (61) and (62), we obtain the entropy:

$$
S=-\ln 2+\frac{v+1}{2} \ln (v+1)-\frac{v-1}{2} \ln (v-1) .
$$

Thus, $S$ depends on $Q, P, \Delta Q, \Delta P$ only via $v$. We have

$$
\frac{d S}{d v}=\frac{1}{2} \ln \frac{v+1}{v-1}>0,
$$

so that $S$ is an increasing function of $v$. Near $v=1$,

$$
S \approx-\frac{v-1}{2} \ln (v-1)
$$

Asymptotically $(v \rightarrow \infty)$,

$$
S \approx \ln v+1-\ln 2 .
$$

In the classical region, $v \gg 1, S \approx \ln v$.

It is clear that the choice of $\mathrm{Q}$ and $\mathrm{P}$ cannot influence the entropy. The independence of $S$ from $Q$ and $P$ does not contradict the Legendre transformation properties. Indeed, usually, one would have

$$
\frac{\partial S}{\partial Q}=\lambda_{1}
$$

but here

$$
\frac{\partial S}{\partial Q}=\lambda_{1}+2 \lambda_{3} Q
$$

which is zero.

The state operator can also be expressed in terms of the averages and variances. The trivial generalization to $n$ degrees of freedom is

Theorem 2 The state operator of the ME-packet of a system with given averages and variances $Q_{1}, \ldots, Q_{n}, \Delta Q_{1}, \ldots, \Delta Q_{n}$ of coordinates and $P_{1}, \ldots, P_{n}$, $\Delta P_{1}, \ldots, \Delta P_{n}$ of momenta, is

$$
\rho=\prod_{k=1}^{n}\left[\frac{2}{v_{k}^{2}-1} \exp \left(-\frac{1}{\hbar} \ln \frac{v_{k}+1}{v_{k}-1} K_{k}\right)\right],
$$


where

$$
K_{k}=\frac{1}{2} \frac{\Delta P_{k}}{\Delta Q_{k}}\left(q_{k}-Q_{k}\right)^{2}+\frac{1}{2} \frac{\Delta Q_{k}}{\Delta P_{k}}\left(p_{k}-P_{k}\right)^{2}
$$

and

$$
v_{k}=\frac{2 \Delta P_{k} \Delta Q_{k}}{\hbar}
$$

Strictly speaking, the state operator (64) is not a Gaussian distribution. Thus, it seems to be either a counterexample to, or a generalization of, Jaynes statement that the Gaussian distribution is the only distribution that maximizes entropy for given values of the first two moments [22].

In the diagonal representation, we have

$$
\rho=\sum_{k=0}^{\infty} R_{k}|k\rangle\langle k|
$$

We easily obtain for $R_{k}$ that

$$
R_{k}=2 \frac{(v-1)^{k}}{(v+1)^{k+1}}
$$

Hence,

$$
\lim _{\nu=1} R_{k}=\delta_{k 0},
$$

and the state $\rho$ becomes $|0\rangle\langle 0|$. In general, states $|k\rangle$ depend on $v$. The state vector $|0\rangle$ in the $q$-representation expressed as a function of $Q, P, \Delta Q$ and $v$ is given by

$$
\psi(q)=\left(\frac{1}{\pi} \frac{v}{2 \Delta Q^{2}}\right)^{1 / 4} \exp \left[-\frac{v}{4 \Delta Q^{2}}(q-Q)^{2}+\frac{i P q}{\hbar}\right] .
$$

This is a Gaussian wave packet that corresponds to different values of variances than the original ME packet but has the minimal uncertainty. For $v \rightarrow 1$, it remains regular and the projector $|0\rangle\langle 0|$ becomes the state operator of the original ME packet. Hence, Gaussian wave packets are special cases of ME-packets.

The diagonal representation offers a method for calculating averages of coordinates and momenta products that replaces the partition function way. Let us denote such a product $X$. We have

$$
\langle X\rangle=\sum_{k=0}^{\infty} R_{k}\langle k|X| k\rangle .
$$

To calculate $\langle k|X| k\rangle$, we use (55), (56), (53), (54), (61) and (62) to obtain

$$
q=Q+\frac{\Delta Q}{\sqrt{v}}\left(A+A^{\dagger}\right), \quad p=P-i \frac{\Delta P}{\sqrt{v}}\left(A-A^{\dagger}\right) .
$$


By substituting these relations to $X$ and using the commutation relations $\left[A, A^{\dagger}\right]=1$, we obtain

$$
X=\mathcal{P}(N)+\mathcal{Q}\left(A, A^{\dagger}\right),
$$

where $N=A^{\dagger} A$ and where, in each monomial of the polynomial $\mathcal{Q}$, the number of $A$-factors is different from the number of $A^{\dagger}$-factors. Thus,

$$
\langle k|X| k\rangle=\mathcal{P}(k) .
$$

In (70), there are, therefore, sums

$$
\sum_{k=0}^{\infty} k^{n} R_{k}
$$

With (68), this becomes

$$
\sum_{k=0}^{\infty} k^{n} R_{k}=\frac{2}{v+1} I_{n},
$$

where

$$
I_{n}(v)=\sum_{k=0}^{\infty} k^{n}\left(\frac{v-1}{v+1}\right)^{k}
$$

We easily obtain

$$
I_{n}=\left(\frac{v^{2}-1}{2} \frac{d}{d v}\right)^{n} \frac{v+1}{2} .
$$

The desired average value is then given by

$$
\langle X\rangle=\frac{2}{v+1} \mathcal{P}\left(\frac{v^{2}-1}{2} \frac{d}{d v}\right) \frac{v+1}{2} .
$$

The calculation of the polynomial $\mathcal{P}$ for a given $X$ and the evaluation of the righthand side of (71) are the two steps of the promised method.

\subsection{Equations of Motion}

Let the Hamiltonian of our system be $H$ and the unitary evolution group $U(t)$. The dynamics in the Schrödinger picture leads to the time dependence of $\rho$ :

$$
\rho(t)=U(t) \rho U^{\dagger}(t) .
$$

Substituting for $\rho$ from (64) and using a well-known property of exponential function, we obtain

$$
\rho(t)=\frac{2}{v^{2}-1} \exp \left(-\frac{1}{\hbar} \ln \frac{v+1}{v-1} U(t) K U^{\dagger}(t)\right) .
$$


In the Heisenberg picture, $\rho$ remains constant, while $q$ and $p$ are time dependent and satisfy the equations

$$
i \hbar \frac{d q}{d t}=[q, H], \quad i \hbar \frac{d p}{d t}=[p, H]
$$

They are solved by

$$
q(t)=U^{\dagger}(t) q U(t), \quad p(t)=U^{\dagger}(t) p U(t),
$$

where $q$ and $p$ are the initial operators, $q=q(0)$ and $p=p(0)$. The resulting operators can be written in the form of operator functions analogous to classical expressions (13) so that (15) and (16) can again be used.

The example with potential function (17) is solvable in quantum theory, too, and we can use it for comparison with the classical dynamics as well as for a better understanding of the ME-packet dynamics. Equation (73) have then the solutions given by (18) and (19) with functions $f_{n}(t)$ and $g_{n}(t)$ given by (20) and (21) or (22) and (23). The calculation of the averages and variances is analogous to the classical one and we obtain (24) and (25) again with the difference that the term $2\langle q p\rangle$ on the right hand side of (25) is now replaced by $\langle q p+p q\rangle$.

To calculate $\langle q p+p q\rangle$, we use the method introduced in the previous section. We have

$$
\begin{aligned}
q p+p q= & 2 Q P+2 \frac{P \Delta Q}{\sqrt{v}}\left(A+A^{\dagger}\right) \\
& -2 i \frac{Q \Delta P}{\sqrt{v}}\left(A-A^{\dagger}\right)-2 i \frac{\Delta Q \Delta P}{v}\left(A^{2}-A^{\dagger 2}\right),
\end{aligned}
$$

hence, $\mathcal{P}=2 Q P$, and

$$
\langle q p+p q\rangle=2 Q P .
$$

The result is again (26). Similarly for $p$, the results are given by (27) and (28).

We have shown that the averages and variances of quantum ME-packets have exactly the same time evolution as those of classical ME-packets in the special case of at-most-quadratic potentials. From formulae (26) and (28) we can also see an interesting fact. On the one hand, both variances must increase near $t=0$. On the other, the entropy must stay constant because the evolution of the quantum state is unitary. As the relation between entropy and $v$ is fixed for ME-packets, the ME-packet form is not preserved by the evolution (the entropy ceases to be maximal). This is similar for Gaussian-packet form or for coherent-state form.

For general potentials, there will be two types of corrections to the dynamics of the averages: terms containing the variances and terms containing $\hbar$. To see these corrections, let us calculate time derivatives for the Hamiltonian (11) with potential (29). The Heisenberg-picture equations of motion give again

$$
\frac{d q}{d t}=\frac{1}{m} p
$$


so that (30) is valid. The other equation,

$$
i \hbar \frac{d p}{d t}=[p, H]
$$

can be applied iteratively as in the classical case so that all time derivatives of $p$ can be obtained. Thus,

$$
\frac{d p}{d t}=-V_{1}-V_{2} q-\frac{V_{3}}{2} q^{2}-\frac{V_{4}}{6} q^{3}+r_{5}
$$

and

$$
\frac{d^{2} p}{d t^{2}}=-\frac{V_{2}}{m} p-\frac{V_{3}}{2 m}(q p+p q)-\frac{V_{4}}{6 m}\left(q^{2} p+q p q+p q^{2}\right)+r_{5} .
$$

This differs from the classical equation only by factor ordering. We can use the commutator $[q, p]=i \hbar$ to simplify the last term,

$$
\frac{d^{2} p}{d t^{2}}=-\frac{V_{2}}{m} p-\frac{V_{3}}{2 m}(q p+p q)-\frac{V_{4}}{2 m} q p q+r_{5} .
$$

Similarly,

$$
\begin{aligned}
\frac{d^{3} p}{d t^{3}}= & -\frac{V_{3}}{m^{2}} p^{2}-\frac{V_{4}}{m^{2}} p q p+\frac{V_{1} V_{2}}{m} \\
& +\frac{V_{1} V_{3}+V_{2}^{2}}{m} q+\frac{3 V_{2} V_{3}+V_{1} V_{4}}{2 m} q^{2} \\
& +\frac{4 V_{2} V_{4}+3 V_{3}^{2}}{6 m} q^{3}+\frac{5 V_{3} V_{4}}{12 m} q^{4}+\frac{V_{4}^{2}}{12 m} q^{5}+r_{5},
\end{aligned}
$$

and

$$
\begin{aligned}
\frac{d^{4} p}{d t^{4}}= & -\frac{V_{4}}{m^{3}} p^{3}+\frac{3 V_{1} V_{3}+V_{2}^{2}}{m^{2}} p \\
& +\frac{3 V_{1} V_{4}+5 V_{2} V_{3}}{2 m^{2}}(q p+p q)+\frac{5 V_{3}^{2}+8 V_{2} V_{4}}{2 m^{2}} q p q \\
& +\frac{3 V_{3} V_{4}}{2 m^{2}}\left(q^{3} p+p q^{3}\right)+\frac{3 V_{4}^{2}}{4 m^{2}} q^{2} p q^{2}+r_{5} .
\end{aligned}
$$

Next, we calculate quantum averages with the help of formula (71). The quantum averages of the monomials that are linear in one of variables $q$ or $p$ can differ from their classical counterparts only by terms that are of the first order in $1 / v$ and purely imaginary. For example,

$$
\langle q p\rangle=Q P+\frac{i \hbar}{2}
$$

or

$$
\left\langle q^{3} p\right\rangle=Q^{3} P+3 Q P \Delta Q^{2}+3 i \frac{Q^{2} \Delta Q \Delta P}{v}+3 i \frac{\Delta Q^{3} \Delta P}{v} .
$$


These corrections clearly cancel for all symmetric factor orderings. The first term in which a second-order correction occurs is $q^{2} p^{2}$ and we obtain for it:

$$
\left\langle p q^{2} p\right\rangle=\left\langle q^{2} p^{2}\right\rangle_{\text {class }}+2 \frac{\Delta Q^{2} \Delta P^{2}}{v^{2}} .
$$

Equations (74)-(77) do not contain any such terms and so their averages coincide exactly with the classical equations (35)-(38). The terms $q^{2} p^{2}$ with different factor orderings occur in the fifth time derivative of $p$ and have the form

$$
\frac{3 V_{3} V_{4}}{2 m^{2}}\left[q^{3} p+p q^{3}, \frac{p^{2}}{2 m}\right]+\frac{V_{3} V_{4}}{2 m^{3}}\left[\frac{1}{3} q^{3}, p^{3}\right]=i \hbar \frac{V_{3} V_{4}}{2 m^{3}}\left(21 p q^{2} p-11 \hbar^{2}\right) .
$$

The average of the resulting term in the fifth time derivative of $p$ is

$$
\frac{V_{3} V_{4}}{2 m^{3}}\left(21 Q^{2} P^{2}+21 P^{2} \Delta Q^{2}+21 Q^{2} \Delta P^{2}+21 \Delta Q^{2} \Delta P^{2}-\frac{\hbar^{2}}{2}\right) .
$$

If we express $\hbar$ as $2 \Delta Q \Delta P / \nu$, we can write the last two terms in the parentheses as

$$
\Delta Q^{2} \Delta P^{2}\left(21-\frac{2}{v^{2}}\right)
$$

A similar term appears in the third time derivative of $p$, if we allow $V_{5} \neq 0$ in the expansion (29):

$$
\left[-\frac{V_{5}}{12 m}\left(q^{3} p+p q^{3}\right), \frac{p^{2}}{2 m}\right]=i \hbar\left[-\frac{V_{5}}{4 m^{2}}\left(2 p q^{2} p+\hbar^{2}\right)\right],
$$

which contributes to $d^{3} P / d t^{3}$ by

$$
-\frac{V_{5}}{2 m^{2}}\left(\left\langle q^{2} p^{2}\right\rangle_{\text {class }}+\frac{4 \Delta Q^{2} \Delta P^{2}}{v^{2}}\right) .
$$

Again, the correction is of the second order in $v^{-1}$.

We can conclude. The quantum equations begin to differ from the classical one's only in the higher order terms in $V$ or in the higher time derivatives and the correction is of the second order in $1 / v$. This seems to be very satisfactory: our quantum model reproduces the classical dynamic very well. Moreover, (69) shows that Gaussian wave packets are special cases of ME packets with $v=1$. Thus, they approximate classical trajectories less accurately than ME packets with large $v$.

\section{Classical Limit}

At some places of the paper, it is written that $v \gg 1$ is the classical regime. Let us now look to see if our equations give some support to this statement.

Let us consider averages of powers of $q$ and $p$. If we expand such an average in powers of Planck constant then the leading term can be calculated with help of the 
formula (10) from quantum partition function (60) and from relations (61) and (62) between the Lagrange multipliers and the averages and variances of $q$ and $p$. This has been explained after the proof of Lemma 2.

The quantum partition function (60) differs from its classical counterpart (6) by the denominator $\sinh \left(\hbar \sqrt{\lambda_{3} \lambda_{4}}\right)$. If

$$
\hbar \sqrt{\lambda_{3} \lambda_{4}} \ll 1
$$

we can write

$$
\sinh \left(\hbar \sqrt{\lambda_{3} \lambda_{4}}\right)=\hbar \sqrt{\lambda_{3} \lambda_{4}}\left[1+O\left(\left(\hbar \sqrt{\lambda_{3} \lambda_{4}}\right)^{2}\right)\right]
$$

The leading term in the partition function then is

$$
Z=\frac{\pi}{h} \frac{1}{\sqrt{\lambda_{3} \lambda_{4}}} \exp \left(\frac{\lambda_{1}^{2}}{4 \lambda_{3}}+\frac{\lambda_{2}^{2}}{4 \lambda_{4}}\right),
$$

where $h=2 \pi \hbar$. Comparing this with formula (6) shows that the two expressions are identical, if we set

$$
v=h .
$$

We can say that quantum mechanics gives us the value of $v$. Next, we have to express condition (78) in terms of the averages and variances. Equations (62) imply

$$
\hbar \sqrt{\lambda_{3} \lambda_{4}}=\frac{1}{2} \ln \frac{v+1}{v-1} .
$$

Hence, condition (78) is equivalent to

$$
v \gg 1 \text {. }
$$

The expression

$$
\frac{v}{2} \ln \frac{v+1}{v-1}
$$

that appears on the right-hand sides of (61) and (62) satisfies

$$
\lim _{\nu=\infty} \frac{v}{2} \ln \frac{v+1}{v-1}=1
$$

Hence, the leading terms in these equations coincide with (7) and (8).

Our result can be formulated as follows. The time evolution of classical and quantum ME packets with the same initial values of averages and variances defines the averages as time functions. These time functions coincide for the two theories in the limit $v=\infty$. Hence, in our approach, this is the classical limit. It is very different from the usual assumption that the classical limit must yield the variances as small as possible. One also often requires that commutators of observables vanish in classical limit. This is however only motivated by the assumption that all basic quantum properties are single values of observables. Within our interpretation, this assumption is rejected and if classical observables are related to quantum operators then only by 
being average values of the operators in prepared states. All such averages are defined by the preparation and do exist simultaneously, independently of whether the operators commute or not. For example, $Q$ and $P$ are such simultaneously existing variables for ME packets.

Let us compare the present paper notion of classical limit with a modern textbook version such as Chap. 14 of [24]. Both approaches define the classical limit of a quantum state as a classical ensemble described by a fuzzy distribution function and calculate time evolutions of averages in the states. However, in the textbook, any quantum system, even not macroscopic, and any state, even pure, are allowed (pure states preferred as they have smaller uncertainties). Hence, our notion is much narrower: we consider only macroscopic quantum systems and only some of their maximum entropy states. This has obvious physical reasons explained in the first two sections.

\section{Conclusion}

The paper describes a quite general construction of quantum states that model important properties of classical-mechanical states. To achieve that, one often-assumed classical property has to be abandoned: the completely sharp trajectory of all mechanical systems. The sharp trajectory is considered here only as an ideal limit allowed by classical mechanics. There is however nothing in nature that corresponds to it. This is in agreement both with practical observations and with theoretical idea that the correct underlying theory is quantum mechanics. Hence, the way for statistical methods highlighted in Ref. [4] is free. The key concept turned out to be physical conditions equivalent to preparation process in quantum mechanics. The paper transfers it into classical mechanics, where it generalizes the old notion of initial data. Entropy is defined by the physical conditions independently of the state of any observer's mind.

Classical mechanics allows not only sharp, but also fuzzy trajectories and the comparison of some classical and quantum fuzzy trajectories shows a very good match. The fuzzy states chosen here are the so-called ME packets. Their fuzziness is described by the quantity $v=2 \Delta Q \Delta P / \hbar$. The entropy of an ME packet depends only on $v$ and is an increasing function of it. The larger $v$ is, the better the quantum and the classical evolutions of average values have been shown to agree. Thus, the classical regime is neither $\Delta Q=\Delta P=0$ (absolutely sharp trajectory) nor $v=1$ (minimum quantum uncertainty). This is the most important result of the paper.

Unlike internal classical properties the external ones such as coordinates and momenta are well manipulable so that conditions exist allowing ME packets to have all variances $\Delta Q$ and $\Delta P$ from a broad range. There is no reason why the variances had always to be small other than the incorrect assumption that all real mechanical trajectories are absolutely sharp. Hence, our account of classical properties as statistical properties of macroscopic quantum systems that started in [4] can be considered as concluded.

Acknowledgements The author is indebted to Ferenc Niedermayer and Jiri Tolar for discussions. 


\section{References}

1. Zurek, W.H.: Rev. Mod. Phys. 75, 715 (2003)

2. Giulini, D., Joos, E., Kiefer, C., Kupsch, J., Stamatescu, I.-O., Zeh, H.D.: Decoherence and the Appearance of Classical World in Quantum Theory. Springer, Berlin (1996)

3. Bub, J.: Interpreting the Quantum World. Cambridge University Press, Cambridge (1997)

4. Hájíček, P., Tolar, J.: Intrinsic properties of quantum systems, arXiv:0806.4437

5. d'Espagnat, B.: Veiled Reality. Addison-Wesley, Reading (1995)

6. Peres, A.: Quantum Theory: Concepts and Methods. Kluwer Academic, Dordrecht (1995)

7. Poulin, D.: Phys. Rev. A 71, 022102 (2005)

8. Kofler, J., Brukner, Č.: Phys. Rev. Lett. 99, 180403 (2007)

9. Hepp, K.: Helv. Phys. Acta 45, 237 (1972)

10. Bell, J.S.: Helv. Phys. Acta 48, 93 (1975)

11. Bóna, P.: Acta Phys. Slov. 23, 149 (1973)

12. Bóna, P.: Acta Phys. Slov. 25, 3 (1975)

13. Bóna, P.: Acta Phys. Slov. 27, 101 (1977)

14. Sewell, G.L.: Quantum Mechanics and its Emergent Macrophysics. Princeton University Press, Princeton (2002)

15. Primas, H.: Asymptotically disjoint quantum states. In: Blanchard, Ph. et al. (eds.) Decoherence: Theoretical, Experimental, and Conceptual Problems. LNP, vol. 538. Springer, Berlin (2002)

16. Bell, J.S.: Against 'measurement'. In: Miller, A.I. (ed.) Sixty Two Years of Uncertainty. Plenum, New York (1990)

17. Wallace, D.: The quantum measurement problem: state of play, arXiv:0712.0149v1 [quant-ph]

18. Gemmer, J., Michel, M., Mahler, G.: Quantum Thermodynamics. Emergence of Thermodynamic Behaviour Within Composite Quantum Systems. LNP, vol. 657. Springer, Berlin (2004)

19. Linden, N., et al.: Quantum mechanical evolution towards thermal equilibrium, arXiv:0812.2385

20. Goldstein, S., et al.: Phys. Rev. Lett. 96, 050403 (2006)

21. Isham, C.J.: Lectures on Quantum Theory. Mathematical and Structural Foundations. Imperial College Press, London (1995)

22. Jaynes, E.T.: Probability Theory. The Logic of Science. Cambridge University Press, Cambridge (2003)

23. von Neumann, J.: Mathematical Foundation of Quantum Mechanics. Princeton University Press, Princeton (1983)

24. Ballentine, L.E.: Quantum Mechanics. A Modern Development. World Scientific, Singapore (2008) 